\title{
Clinical trial registration in physiotherapy journals: recommendations from the International Society of Physiotherapy Journal Editors
}

Clinical trial registration involves placing the protocol for a clinical trial on a free, publicly available and electronically searchable register. Registration is considered to be prospective if the protocol is registered before the trial commences (i.e., before the first participant is enrolled). Prospective registration has several potential advantages. It could help avoid trials being duplicated unnecessarily and it could allow people with health problems to identify trials in which they might participate. Perhaps more importantly, however, it tackles two big problems in clinical research: selective reporting and publication bias.

Selective reporting involves investigators only reporting the most favourable results when they publish a trial, instead of reporting the results for all the outcomes that were measured. Reporting only favourable outcomes can create a misleading appearance of the effect of a therapy in the published literature. For example, imagine that a completely ineffective intervention is tested across several trials and each trial measures multiple outcomes. Most outcomes will show no significant effect of the intervention. However, occasionally an outcome will show significant benefit or harm simply by chance. If the researchers publish the positive outcomes but not all of the non-significant and negative outcomes, readers could interpret falsely that the intervention is beneficial. A similar problem could occur when outcomes are analysed at multiple time points. Researchers may report that an intervention improves walking speed at 6 months, but fail to mention that it does not improve walking speed at 1, 2, 3, 9, 12 and 24 months. Prospective registration of clinical trials combats this problem in several ways. Journal editors and reviewers can compare the range of outcomes reported in a manuscript against those listed in the registered protocol, requesting that any discrepancies be resolved by following the protocol. Readers can also compare the outcomes in the registered protocol against those in the published report, taking greater reassurance when they are consistent.
Publication bias arises when trials with positive results are more likely to be published than trials with non-significant or negative results. Like selective reporting, this can also spuriously inflate the apparent effect of an intervention across the published data. For example, a trial in which the intervention appeared to be effective may be published, while the three other trials in which the intervention appeared ineffective or harmful languish in the filing cabinets of the investigators. If a trial is registered but never published, authors of a systematic review can still find the trial on the register and contact the authors to request the unpublished data for inclusion in the review. Therefore, prospective registration of clinical trials could further limit bias affecting the body of evidence that is available in published physiotherapy trials.

Prospective clinical trial registration encourages transparency ${ }^{1}$ and may also make it more difficult for fraudulent authors to fabricate data. For example, some journals now ask for individual patient data to be provided routinely for checking ${ }^{2}$ or audit data when fraud is suspected ${ }^{3}$. Data collection should have occurred during the dates of data collection defined on the registry. Because many outcomes are measured and stored electronically with date stamps, this would increase the planning and complexity involved in fabricating data, especially if the fabricated data are to withstand the scrutiny of an audit. Also, researchers who obtain unwelcome data from a particular subgroup of patients may be tempted to eliminate it by retrospectively introducing an additional exclusion criterion. If their protocol has been prospectively registered, however, this would be publicly evident to anyone who compared the registered protocol and the report of the trial.

\section{How common is clinical trial registration?}

The first major register for healthcare trials was established in $1998^{4}$. Although thousands of trials were soon registered, the majority of trials remained 
unregistered. In 2004, clinical trial registration was endorsed by the International Committee of Medical Journal Editors (ICMJE) ${ }^{4}$. In addition to endorsing clinical trial registration, member journals of the ICMJE made prospective registration compulsory for all clinical trials that commenced participant recruitment after 1 July $2005^{4}$. Many other journals also endorsed clinical trial registration and the number of registered trials increased rapidly ${ }^{5}$. Since then, many organisations have added their support for clinical trial registration. For example, in 2008 the World Medical Association included a new item on the Declaration of Helsinki stating that 'Every clinical trial must be registered in a publicly accessible database before recruitment of the first subject $\left(\right.$ p.3) ${ }^{6}$. Some ethics committees have made trial registration a condition of ethical approval.

Although some physiotherapy journals have also encouraged clinical trial registration ${ }^{7,8,9}$, only about $6 \%$ of the randomised trials investigating the effects of physiotherapy interventions published in 2009 had been registered prospectively ${ }^{10}$. In an attempt to rectify this situation, this editorial recommending prospective registration has been co-authored by several members of the International Society of Physiotherapy Journal Editors (ISPJE). The remainder of the editorial will: define which trials should be registered; explain how researchers can register their trials; announce tougher policies about clinical trial registration that are being adopted by some member journals of the ISPJE; and identify who can contribute to ensuring that clinical trial registration achieves its potential benefits.

\section{Which trials should be registered?}

Any clinical trial should be prospectively registered before the first participant is recruited into the study. The World Health Organization defines clinical trials as 'any research study that prospectively assigns human participants or groups of humans to one or more health-related interventions to evaluate the effects on health outcomes' ${ }^{11}$.

\section{How can I register my trial?}

Clinical trial registration should be quick, easy and free of charge. Many clinical trial registries have been established, including some that focus on a particular disease (eg, Internet Stroke Center Trials Registry, www.strokecenter.org/trials) or geographical region (eg, Pan African Clinical Trials Registry, www.pactr. org). Researchers often choose to register their trials in their country's national register, although this is not compulsory. It is more important that researchers choose a registry that elicits and documents all the relevant content from the original protocol (outlined below) and that has satisfactory quality, validity, accessibility, unique identification, technical capacity and administration. To assist researchers, the World Health Organization maintains a list of registries that meet these criteria (http://www.who.int/ictrp/network/ primary/en/index.html). Currently 16 registries are listed. Among these, researchers could choose one that processes applications swiftly or that allows communication using their native language.

When registering their protocol, researchers will be asked to provide information such as descriptions of the intervention(s) and comparison(s) studied, study hypotheses, primary and secondary outcomes, eligibility criteria, sample size, blinding, funding, principal investigators and dates of commencement and anticipated completion of the study. It is common for trial registries to review the information for completeness and clarity, so some editing might be needed. The registry will then provide a unique trial registration number to the researchers. This number should be included in all reports of the trial's results as a link to the registered protocol for editors, reviewers and readers.

Prospective registration can be done any time before the first participant is recruited. Many researchers wait until immediately before recruitment starts, so that any late changes to the protocol (such as alterations requested by an ethics committee) do not necessitate an amendment to the registry entry. Although not ideal, protocol amendments are sometimes made after recruitment starts. These should be updated on the registered protocol as well. The trial registry will publicly document what changed and on what date.

\section{ISPJE member journals introducing mandatory prospective registration policies}

The executive of the ISPJE strongly recommends that member journals adopt a policy of mandatory prospective registration for all clinical trials. Several member journals are implementing such policies. Physical Therapy has already implemented a policy of mandatory prospective clinical trial registration, which applies to trials that commenced participant recruitment after 1 January 2009. The following table lists other member journals and their nominated dates to implement mandatory prospective clinical trial registration, as well as the trials that this policy applies to (based on the commencement date of participant recruitment).

\section{Who else can help ensure clinical trial registration achieves its potential benefits?}

In addition to the recommendations for researchers and editorial boards outlined above, others can contribute to ensuring that clinical trial registration achieves its potential benefits. Everyone in the profession can ensure that their colleagues are aware of clinical trial registration and its importance. 


\begin{tabular}{|c|c|c|}
\hline Journal & $\begin{array}{l}\text { Date after which } \\
\text { prospective clinical } \\
\text { trial registration } \\
\text { becomes mandatory }\end{array}$ & $\begin{array}{l}\text { This policy applies } \\
\text { to trials that commence } \\
\text { participant recruitment } \\
\text { after this date }\end{array}$ \\
\hline Brazilian Journal of Physical Therapy/Revista Brasileira de Fisioterapia & 1 January 2014 & 31 December 2013 \\
\hline Cardiopulmonary Physical Therapy Journal & 1 January 2015 & 1 January 2014 \\
\hline Journal of Manual and Manipulative Therapy & 1 January 2014 & 6 June 2013 \\
\hline Journal of Physiotherapy & 1 January 2013 & 1 January 2006 \\
\hline Journal of Physiotherapy \& Sports Medicine & 1 January 2014 & 1 June 2013 \\
\hline Manual Therapy & 1 January 2014 & 1 June 2013 \\
\hline Physical Therapy \& Research/Fisioterapia e Pesquisa & 1 January 2014 & 31 December 2013 \\
\hline Physiotherapy & 1 January 2013 & 1 January 2013 \\
\hline Physiotherapy Canada & 1 January 2013 & 1 January 2013 \\
\hline The Journal of Orthopaedic \& Sports Physical Therapy & 1 January 2013 & 1 January 2013 \\
\hline Tidsskriftet Fysioterapeuten/Norwegian Journal of Physiotherapy & 1 January 2014 & 1 July 2013 \\
\hline
\end{tabular}

Educators should ensure that the research component of physiotherapy training programs explains the importance of trial registration. Clinicians can also advise or help patients to search trial registers to identify relevant trials for which the patient might volunteer. Administrators of clinical trial registries that do not meet the WHO criteria can strive to attain this status. Grant review panels can make funding contingent upon prospective registration for proposed clinical trials. More ethics review committees can make their approval of trials contingent upon prospective registration as well. However, even universal prospective registration may make no difference to selective reporting and publication bias unless there is an expectation that protocols will be compared to published reports before publication. Therefore, journal editors and peer reviewers must remember to check for discrepancies between submitted manuscripts and registry entries.

Physiotherapy clinical trials that are conducted and reported according to a pre-specified protocol are more likely to provide credible information than those that do not. Prospective clinical trial registration is therefore of great potential value to the clinicians, consumers and researchers who rely upon clinical trial data and that is why ISPJE is recommending that members enact a policy for prospective trial registration.

\section{International Society of Physiotherapy Journal Editors}

Leonardo O P Costa and Chung-Wei Christine Lin

\section{Brazilian Journal of Physical Therapy/Revista Brasileira de Fisioterapia}

Debora Bevilaqua Grossi and Marisa Cota Mancini

\section{Cardiopulmonary Physical Therapy Journal}

Anne K Swisher

\author{
Journal of Manual and Manipulative Therapy \\ Chad Cook and Dan Vaughn
}

\section{Journal of Physiotherapy \\ Mark R Elkins \\ Journal of Physiotherapy \& Sports Medicine \\ Umer Sheikh}

\section{Manual Therapy}

Ann Moore and Gwendolen Jull

\section{Physical Therapy}

Rebecca L Craik and Christopher G Maher

Physical Therapy \& Research/Fisioterapia e Pesquisa

Rinaldo Roberto de Jesus Guirro and Amélia Pasqual Marques

\section{Physiotherapy}

Michele Harms

\section{Physiotherapy Canada \\ Dina Brooks}

The Journal of Orthopaedic \& Sports Physical Therapy

Guy G Simoneau

Tidsskriftet Fysioterapeuten/Norwegian Journal of Physiotherapy

John Henry Strupstad

\section{References}

1 Sim I, Chan AW, Gulmezoglu AM, Evans T, Pang T. Clinical trial registration: transparency is the watchword. Lancet. 2006;367:1631-1633.

2 Herbert RD. Researchers should make data freely available. Aust J Physiother. 2008;54:3.

3 Smith J, Godlee F. Investigating allegations of scientific misconduct - Journals can do only so much; institutions need to be willing to investigate. BMJ. 2005;331:245-246.

4 de Angelis C, Drazen JM, Frizelle FA, et al. Clinical trial registration: a statement from the International Committee of Medical Journal Editors. N. Engl. J. Med. 2004;351:1250-1251.

5 Laine C, Horton R, DeAngelis CD, et al. Clinical trial registration - Looking back and moving ahead. N. Engl. J. Med. 2007;356:2734-2736.

6 World Medical Association Declaration of Helsinki. Ethical principles for medical research involving human subjects: World Medical Association Declaration of Helsinki. 2008:1-5.

7 Askie L, Ghersi D, Simes J. Prospective registration of clinical trials. Aust J Physiother. 2006;52:237-239. 
8 Costa LO, Maher CG, Moseley AM, Sherrington C, Herbert RD, Elkins MR. Editorial: endorsement of trial registration and the CONSORT statement by the Revista Brasileira de Fisioterapia. Rev Bras Fisioter. 2010;14:V-VI.

9 Harms M. Clinical trial registration. Physiother. 2011;97: 181.
10 Pinto RZ, Elkins MR, Moseley AM, et al. Comparison of registry entries and published reports of randomised trials: an audit of 200 published trials. Phys Ther. Accepted 12/9/2012.

11 World Health Organization. International Clinical Trials Registry Platform (ICTRP) 2012. Available from http:// www.who.int/ictrp/en/Accessed 09/07/ 2012. 